Case Report

\title{
Acute Hemolytic Transfusion Reaction in Group B Recipient Associated with Group A Apheresis Platelet Donor: Case Report and Literature Review
}

\author{
Tracy R. Shachner and Christopher T. Clark (iD \\ Department of Pathology, University of Tennessee Graduate School of Medicine, Knoxville, TN 37920, USA \\ Correspondence should be addressed to Christopher T. Clark; cclark@utmck.edu
}

Received 23 February 2018; Accepted 30 April 2018; Published 24 June 2018

Academic Editor: John B. Kortbeek

Copyright (c) 2018 Tracy R. Shachner and Christopher T. Clark. This is an open access article distributed under the Creative Commons Attribution License, which permits unrestricted use, distribution, and reproduction in any medium, provided the original work is properly cited.

\begin{abstract}
Acute hemolytic transfusion reaction is a known but rare potential adverse event related to platelet transfusion. Most reported cases of platelet-related hemolytic transfusion reaction have resulted from transfusion of platelets from group $\mathrm{O}$ donor to group $\mathrm{A}$ recipient. We identified only one prior case report in the literature of hemolytic transfusion reactions resulting from transfusion of apheresis platelets from group A donor to group B recipient. In that case report, two platelet units were obtained from a single donation and transfused into two separate patients. Both patients exhibited acute hemolytic reactions. The donor is reported to have high anti-B titers, as well as report of probiotic use. We report a case of acute hemolytic reaction in group B recipient following transfusion of apheresis platelets from group A donor with high-titer anti-B but unknown status of probiotic use. This case demonstrates that while low, there still exists potential risk for hemolysis from out-of-group A plasma transfusion.
\end{abstract}

\section{Introduction}

There is currently no requirement for $\mathrm{ABO}$ compatibility for transfusion of platelet units in the United States (US). The AABB (formerly known as the American Association of Blood Banks) only requires transfusion services to have a policy regarding out-of-group components containing significant amount of incompatible $\mathrm{ABO}$ antibodies [1]. Approximately $10 \%$ to $40 \%$ of platelet transfusions in the US are reportedly $\mathrm{ABO}$-incompatible [2]. There appears to be no consensus on a specific method to minimize transfusion of $\mathrm{ABO}$-incompatible plasma contained within platelet units [3-7]. A retrospective analysis revealed an unspecified adverse event in 3 out of 59,933 out-of-group (ABOincompatible) platelet transfusions within the Medicare database, suggesting an overall low risk with out-of-group platelet transfusion [8]. Prior reports of acute hemolysis related to platelet transfusion have been primarily when group $\mathrm{O}$ platelet unit is transfused into group $\mathrm{A}$ recipient [7, 9-11]. Association of donor group A apheresis platelet unit to group B recipient has been reported from a single donor who donated two platelet units in a single donation, transfused to two separate patients, and both patient recipients demonstrating acute hemolysis. The authors report the donor to have high anti-B titers $(16,384$ at both room temperature \{saline\} and IgG phases) in addition to recent probiotic use [12]. We report a rare case of acute hemolysis in a group $\mathrm{B}, \mathrm{Rh}(\mathrm{D})$-positive patient after receiving a single unit of apheresis platelets from a group $A, R h(D)$-positive donor with unknown history of probiotic use.

\section{Case Presentation}

A 53-year-old, blood group $\mathrm{B}, \mathrm{Rh}(\mathrm{D})$-positive female with history of metastatic adenocarcinoma status after chemotherapy treatment presented to the emergency department with abdominal pain, vomiting, and fever. She was admitted for a possible small bowel obstruction and sepsis. Upon her admission, her complete blood count (CBC) revealed a normal platelet count of $186 \times 10^{3}$ (reference range: $130-400 \times 10^{3} / \mathrm{mm}^{3}$ ), low white blood cell count of $2.7 \times 10^{3}$ cells $/ \mathrm{mm}^{3}$ (reference 
range: $4.4-11 \times 10^{3}$ cells $/ \mathrm{mm}^{3}$ ), normal hemoglobin level of $15.9 \mathrm{~g} / \mathrm{dL}$ (reference range: $12-16 \mathrm{~g} / \mathrm{dL}$ ), and a normal hematocrit of $46.6 \%$ (reference range: $37-47 \%$ ). Red blood cell antibody screen was negative. Over the next few days, the patient's platelet count continually decreased, reaching a critical low level of $10 \times 10^{3}$ cells $/ \mathrm{mm}^{3}$ one week after admission. She received a single unit of apheresis platelets from a group $\mathrm{A}, \mathrm{Rh}(\mathrm{D})$-positive donor. The unit initially contained $3.7 \times 10^{11}$ platelets per milliliter $(\mathrm{mL})$ within total volume of $270 \mathrm{~mL}$. The platelet product was suspended in Anticoagulant Citrate Dextrose Solution, Solution A (ACD-A) and transfused on storage day 5 . The red blood cell visual count was reported negative. Approximately ten minutes after the transfusion was started, the patient began to complain of severe lower back pain. The pain was described as 10/10, sharp and stabbing. No other signs or symptoms were reported, including no fever or blood pressure changes. The patient received $135 \mathrm{~mL}(50 \%)$ of the platelet product. The primary provider care team was notified, and the patient observed while receiving normal saline at $100 \mathrm{~mL} /$ hour. No other treatment was initiated. The patient reports the pain $11 / 2$ hours later to be $5 / 10$ and "much better" 2 hours after transfusion. A transfusion reaction work-up was initiated, which revealed a posttransfusion blood sample with visible hemolysis and 1+ positive direct antiglobulin test (C3b and $\mathrm{C} 3 \mathrm{~d}$ positive; IgG negative). No eluate was performed due to absence of detectable IgG on the posttransfusion DAT sample. Pretransfusion blood sample showed no hemolysis, and pretransfusion DAT was negative. Subsequent Gram stain of the platelet unit revealed no organisms seen. Aerobic and anaerobic cultures of the platelet unit after transfusion showed no growth at 5 days. Initial cultures performed by the blood center also reported no growth at 5 days. No medications or fluids were infused with the product. The results of the work-up were consistent with an acute hemolytic transfusion reaction related to an apheresis platelet unit from a group $A, R h(D)$-positive donor to a group $\mathrm{B}, \mathrm{Rh}(\mathrm{D})$-positive recipient. The antibodies (anti-B) from the platelet unit were titered and found to be high-titer at 512 (Table 1). Titer performed following the College of American Pathologists (CAP) tube method "uniform procedure" utilizing 0.9\% normal saline $(\mathrm{NaCl})$. The hemoglobin 14 hours prior to transfusion was $8.9 \mathrm{~g} / \mathrm{dL}$. The hemoglobin 1 hour 15 minutes after transfusion was $7.4 \mathrm{~g} / \mathrm{dL}$. From a transfusion medicine standpoint, the patient remained stable throughout the remainder of her hospital admission. The platelet product was donated by an 18-year-old male and was part of a double platelet unit donation. The companion platelet aliquot was transfused at another institution to a group compatible recipient without reported adverse event. The donor had previously donated a double red cell product with no known associated adverse event. Multiple attempts to contact donor after reaction did not receive a response. History of probiotic use was not reported by the donor or asked at time of donation. Status of probiotic use could not be confirmed after donation.
TABLE 1: Tube method (saline) titer of donor platelet unit with agglutinin reaction strength at room temperature and at $37^{\circ} \mathrm{C}$.

\begin{tabular}{lcc}
\hline Titer & $\begin{array}{r}\text { Room temperature } \\
\text { at } 30 \text { minutes }\end{array}$ & $\begin{array}{c}37^{\circ} \mathrm{C}(\mathrm{IgG}) \\
\text { at } 30 \text { minutes }\end{array}$ \\
\hline 1 & $4+$ & $4+$ \\
2 & $4+$ & $4+$ \\
4 & $4+$ & $4+$ \\
8 & $4+$ & $4+$ \\
16 & $4+$ & $4+$ \\
32 & $4+$ & $4+$ \\
64 & $4+$ & $3+$ \\
128 & $4+$ & $3+$ \\
256 & $3+$ & $2+$ \\
512 & $1+$ & $2+$ \\
1024 & Negative & Negative \\
2048 & Negative & Negative \\
4056 & Negative & Negative \\
\hline
\end{tabular}

\section{Discussion}

Donor antibodies against human leukocyte antigens (HLA) are a major association reported with transfusion-associated acute lung injury (TRALI) [13]. Group AB plasma is considered the universal donor plasma product and is transfused in emergent situations when the blood type of the recipient is unknown. Prior to 2014, male-only plasma was common TRALI risk-reduction strategy. However, use of female-derived $A B$ plasma was still commonly utilized in emergent situations due to low frequency of $\mathrm{AB}$ blood type in the general population and inability to meet the demand for group AB plasma products for emergent use. A 2014 AABB Bulletin reports that postmitigation TRALI risk was 1.8 per million units distributed (99\% male donors) for donor plasma blood groups A, B, and O. However, the preand postmitigation TRALI risk was essentially unchanged for those receiving group $\mathrm{AB}$ plasma (26.3 cases per million units distributed with $60 \%$ male donors and $40 \%$ female donors). The bulletin states that $82 \%$ of plasma-mediated TRALI reported to the American Red Cross (23 cases from 2008 to 2011) involved female donors. As a result, the 2014 AABB Bulletin states that plasma and whole blood from allogeneic donors shall be from males and females who have not been pregnant or from previously pregnant females who have negative test results for HLA antibodies [14]. This resulted in potential significant loss of available AB plasma products, and therefore, the need to transition to emergent use of group A plasma. Transfusion of group A plasma is $\mathrm{ABO}$-compatible for groups $\mathrm{A}$ and $\mathrm{O}$ recipients. However, transfusion of group A plasma is ABO-incompatible for patients of blood groups $\mathrm{AB}$ and $\mathrm{B}$ who in turn have potential risk of reaction due to minor incompatibility. Blood groups $\mathrm{O}$ and $\mathrm{A}$ are the most common blood types. Blood group $\mathrm{AB}$ is approximately $4 \%$ of the general population. Group B is approximately $9 \%$ of Caucasian, 20\% of African American, and 25\% of Asian populations [15]. Therefore, use of emergent group A plasma will be $\mathrm{ABO}$-compatible for the majority of the general population and $\mathrm{ABO}$-incompatible in the minority. Use of emergent A plasma is thought to have 
low risk of hemolysis [3, 16-19]. Although one unit of standard apheresis platelets stored in human plasma contains more than one volume equivalent of a standard unit of plasma, there currently is no requirement regarding $\mathrm{ABO}$ compatibility for platelet transfusion in adults due to short shelf life of platelet products and availability constraints [1]. While it is important to attempt to limit the amount of outof-group plasma patients receive, out-of-group platelet transfusions occur every day in the US without report of hemolytic reactions [2]. This is supportive evidence that emergent use of group A plasma is likely to have low risk. In addition, during hemorrhagic massive transfusion events, the patient is also receiving group $\mathrm{O}$ red blood cells, likely decreasing the relative risk of hemolysis associated with the group A plasma. There is differing practice regarding titering of out-of-group apheresis platelets and group A plasma units for emergent use. There is also great variation in what is considered low- and high-titer $[5,6,16,17,20,21]$. In our case, the donor had anti-B titer of 512 which is above the cutoff for high titer in our laboratory. Since the majority of reports of acute hemolysis related to platelet transfusion are associated with anti-A from group $\mathrm{O}$ apheresis platelets [7, 9-11], our practice is to perform a modified titer for antiA (isohemagglutinins) of group $\mathrm{O}$ apheresis platelet units prior to out-of-group transfusion. We perform a modified titer for anti-A at 250 and if negative for agglutination, the unit is considered low-titer and is available for transfusion to any blood group recipient. If the platelet unit is positive at titer of 250, the unit is designated as high-titer and reserved only for group $\mathrm{O}$ recipients. The modified titer is a tube test method in which 996 microliters of $0.9 \%$ normal saline $(\mathrm{NaCl})$ is placed in a test tube along with 4 microliters of donor plasma from the platelet unit. In addition, separate tubes for positive control (996 microliters saline, 4 microliters anti-A) and negative control (996 microliters saline, 4 microliters anti-B) are made. One drop of $1: 250$ dilution of each sample is transferred into separate test tubes. One drop of type A reagent red blood cells is added to each tube. After 10 minutes incubation at room temperature, the tubes are spun at $3400 \mathrm{rpm}$ for 15 seconds and read for agglutination. When possible, we transfuse $\mathrm{ABO}$-identical apheresis platelet units to our patients in the neonatal intensive care unit. In addition to screening donors for high-titer $\mathrm{ABO}$ antibodies, other suggested strategies to reduce risk of out-of-group platelet transfusion include transfuse ABOidentical platelet units when possible particularly in highrisk patients (neonates, pediatric patients, hematopoietic stem cell transplant, and organ transplant recipients), wash and resuspend platelets in saline, volume reduction, reduce plasma volume of group $\mathrm{O}$ apheresis platelet concentrates to $50 \mathrm{~mL}$, and replace plasma with additive solutions or $\mathrm{AB}$ plasma after washing [22-29]. We utilize group A plasma in emergent situations, but do not titer or set out-of-group A plasma volume limits. The STAT Study reports that the majority of Level 1 trauma center survey respondents maintain group A plasma for immediate available use and $63 \%$ use group A plasma during initial resuscitation phase of trauma patients with unknown $\mathrm{ABO}$ group. This study also reports that $62 \%$ of respondents do not set limits for potential out-of-group A plasma in trauma patients and only $21 \%$ consider anti-B titer in selection of group A plasma units dedicated for emergent use $[19,21]$.

\section{Conclusion}

There is currently no standard practice for performing titers on emergent group A plasma or for out-of-group apheresis platelet products prior to transfusion. While out-of-group apheresis platelet transfusion is acceptable common practice and emergent use of group A plasma is becoming more common, our case report demonstrates that while low, there still exists potential risk of acute hemolytic transfusion reaction from group A plasma transfused to a group B recipient. It would be helpful to have additional well-controlled studies to evaluate the level of significant isohemagglutinin titers for out-ofgroup transfusion and recommendation guidelines for standardized practice. Additional investigation into the potential significance of probiotic use in the donor population could also be beneficial.

\section{Conflicts of Interest}

The authors declare that they have no conflicts of interest.

\section{References}

[1] T. Price, Standards for Blood Banks and Transfusion Services, AABB, Bethesda, MD, USA, 26th edition, 2009.

[2] L. L. Cooling, T. A. Downs, S. H. Butch, and R. D. Davenport, "Anti-A and anti-B titers in pooled group O platelets are comparable to apheresis platelets," Transfusion, vol. 48, pp. 2106-2113, 2008.

[3] J. Cid, S. K. Harm, and M. H. Yazer, "Platelet transfusion-the art and science of compromise," Transfusion Medicine and Hemotherapy, vol. 40, pp. 160-171, 2013.

[4] N. M. Dunbar, D. L. Ornstein, and J. J. Dumont, "ABO incompatible platelets: risks versus benefit," Current Opinion in Hematolology, vol. 19, pp. 475-479, 2012.

[5] C. D. Josephson, M. I. Castillejo, K. Grima, and C. D. Hillyer, "ABO-mismatched platelet transfusion: strategies to mitigate patient exposure to naturally occurring hemolytic antibodies," Transfusion Apheresis Science, vol. 42, pp. 83-88, 2010.

[6] M. K. Fung, K. A. Downes, and I. A. Shulman, "Transfusion of platelets containing ABO-incompatible plasma," Archives of Pathology and Laboratory Medicine, vol. 131, pp. 909-916, 2007.

[7] M. Lozano and J. Cid, "The clinical implication of platelet transfusions associated with ABO or Rh(D) incompatibility," Transfusion Medicine Reviews, vol. 17, no. 1, pp. 57-68, 2003.

[8] M. Menis, H. S. Izurieta, S. A. Anderson et al., "Outpatient transfusion and occurrence of serious noninfectious transfusion-related complications among US elderly, 20072008: utility of large administrative databases in blood safety research," Transfusion, vol. 52, pp. 1968-1976, 2012.

[9] L. Cooling, "ABO and platelet transfusion therapy," Immunohematology, vol. 23, pp. 20-33, 2007.

[10] S. Sapatnekar, S. Girish, K. A. Downes, S. Wiersma, C. McGarth, and R. Yomtovian, "Acute hemolytic transfusion reaction in a pediatric patient following transfusion of 
apheresis platelets," Journal of Clinical Apheresis, vol. 20, pp. 225-229, 2005.

[11] A. Angioliool and N. L. Luban, "Hemolysis following an out-ofgroup platelet transfusion in an 8-month-old with Langerhans cell histiocytosis," Journal Pediatric Hematology Oncology, vol. 26, no. 4, pp. 267-269, 2004.

[12] J. Daniel-Johnson, S. Leitman, H. Klein et al., "Probioticassociated high-titer anti-B in a group A platelet donor as a cause of severe hemolytic transfusion reactions," Transfusion, vol. 49, no. 9, pp. 1845-1849, 2009.

[13] B. H. Shaz, S. R. Stowell, and C. D. Hillyer, "Transfusionrelated acute lung injury: from bedside to bench and back," Blood, vol. 17, no. 5, pp. 1463-1471, 2011.

[14] G. Sher and M. A. Markowitz, "AABB Association Bulletin \#14-02,” February 2014, http://www.aabb.org/programs/ publications/bulletins/Documents/ab14-02.pdf.

[15] M. E. Reid and C. Lomas-Francis, The Blood Group Antigen Facts Book, Elsevier, New York, NY, USA, 2nd edition, 2007.

[16] V. Chhibber, M. Greene, M. Vauthrin, J. Bailey, and R. Weinstein, "Is group A thawed plasma suitable as the first option for emergency release transfusion?," Transfusion, vol. 54, pp. 1751-1754, 2014.

[17] M. D. Zielinski, P. M. Johnson, D. Jenkins, N. Goussous, and J. R. Stubbs, "Emergency use of prethawed Group A plasma in trauma patients," Journal Trauma Acute Care Surgery, vol. 74, no. 1, pp. 69-75, 2012.

[18] W. T. Stevens, B. C. Morse, A. Bernard et al., "Incompatible type A plasma transfusion in patients requiring massive transfusion protocol: outcomes of an Eastern Association for the Surgery of Trauma multicenter study," Journal of Trauma and Acute Care Surgery, vol. 83, no. 1, pp. 25-29, 2017.

[19] N. M. Dunbar, M. H. Yazer, on behalf of the Biomedical Excellence for Safer Transfusion (BEST) Collaborative, and the STAT, "Safety of the use of group A plasma in trauma: the STAT study," Study Investigators Transfusion, vol. 57, no. 8, pp. 1879-1884, 2017.

[20] M. S. Karafin, L. Blagg, A. A. Tobian, K. E. King, P. M. Ness, and W. J. Savage, "ABO antibody titers are not predictive of hemolytic reaction due to plasma-incompatible platelet transfusions," Transfusion, vol. 52, no. 10, pp. 2087-2093, 2012.

[21] N. M. Dunbar and M. H. Yazer, "Biomedical Excellence for Safer Transfusion Collaborative. A possible new paradigm? A survey-based assessment of the use of thawed group A plasma for trauma resuscitation in the United States," Transfusion, vol. 56, no. 1, pp. 125-129, 2015.

[22] A. V. Romphruk, S. Cheunta, L. Pakoate et al., "Preparation of single donor platelet with low antibody titers for all patients," Transfusion and Apheresis Science, vol. 46, pp. 125-128, 2012.

[23] H. Alhumaidan and J. Sweeney, "Current status of additive solutions for platelets," Transfusion and Apheresis Science, vol. 27, pp. 93-98, 2012.

[24] M. J. Fontaine, A. M. Mills, S. Weiss, W. J. Hong, M. Viele, and L. T. Goodnough, "How we treat: risk mitigation for ABO-incompatible plasma in plateletpheresis products," Transfusion, vol. 52, pp. 2081-2085, 2012.

[25] K. Quillen, "Hemolysis form platelet transfusion: call to action for an underreported reaction," Transfusion, vol. 52, pp. 2072-2074, 2012.

[26] K. Quillen, S. L. Sheldon, J. A. Daniel-Johnson, A. H. LeeStroka, and W. A. Flegel, "A practical strategy to reduce the risk of passive hemolysis by screening plateletpheresis donors for high-titer ABO antibodies," Transfusion, vol. 51, pp. 9296, 2011.
[27] P. Perseghin, "High concentration plasma-reduced plateletpheresis concentrates," Transfusion and Apheresis Science, vol. 44, pp. 273-276, 2011.

[28] C. D. Josephson, M. I. Castillejo, K. Grima, and C. D. Hillyer, "ABO-mismatched platelet transfusions: strategies to mitigate patient exposure to naturally occurring hemolytic antibodies," Transfusion and Apheresis Science, vol. 42, pp. 83-88, 2010.

[29] S. Valsami, D. Mimitrios, A. Gialeraki, M. Chimonidou, and M. Politou, "Current trends in platelet transfusion practice: the role of ABO-RHD and human leukocyte antigen incompatibility," Asian Journal of Transfusion Science, vol. 9, no. 2, pp. 117-123, 2015. 


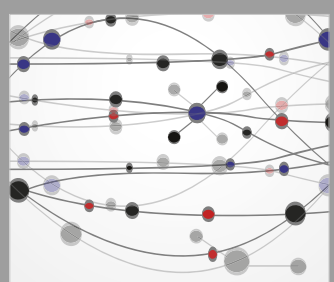

The Scientific World Journal
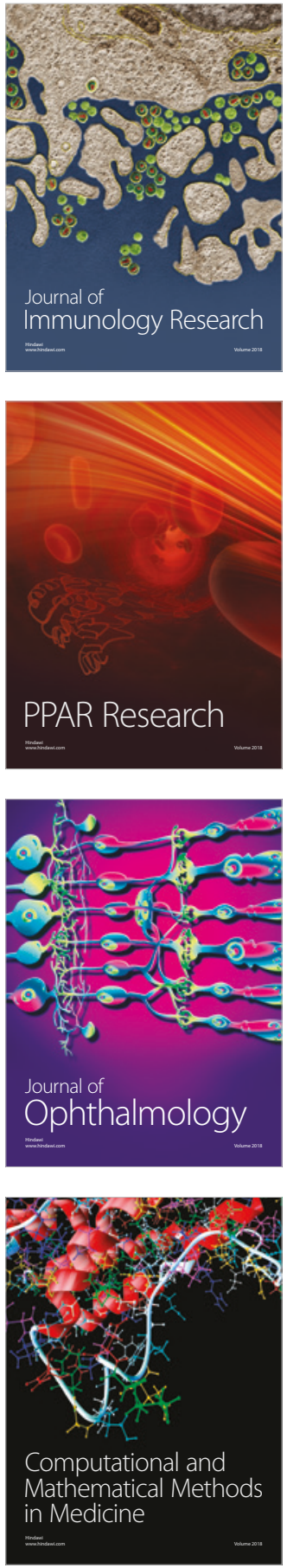

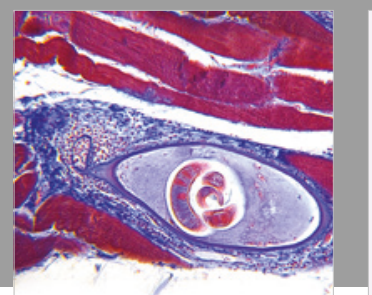

Gastroenterology Research and Practice

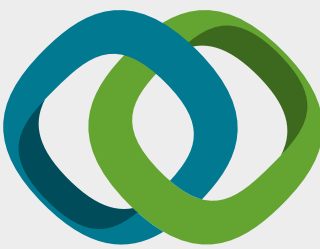

\section{Hindawi}

Submit your manuscripts at

www.hindawi.com
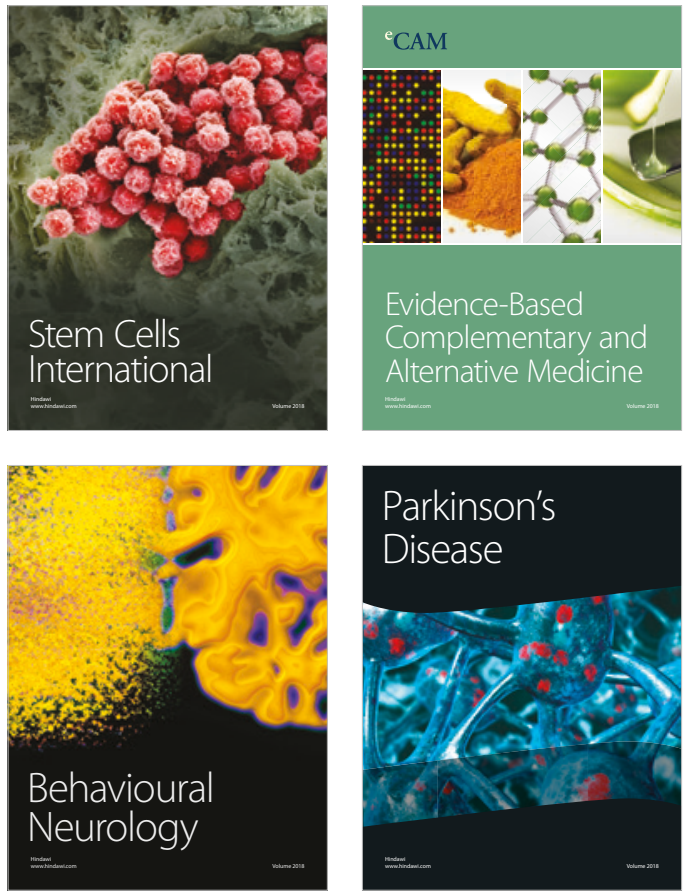

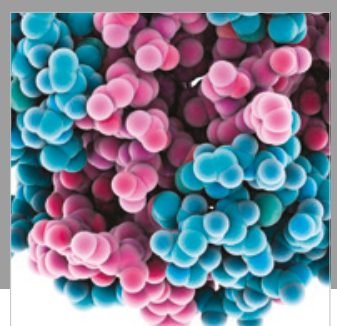

ournal of

Diabetes Research

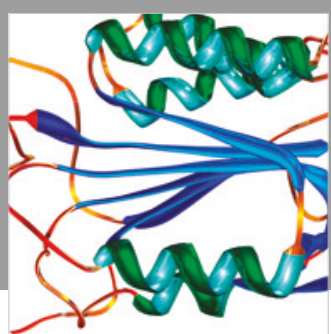

Disease Markers
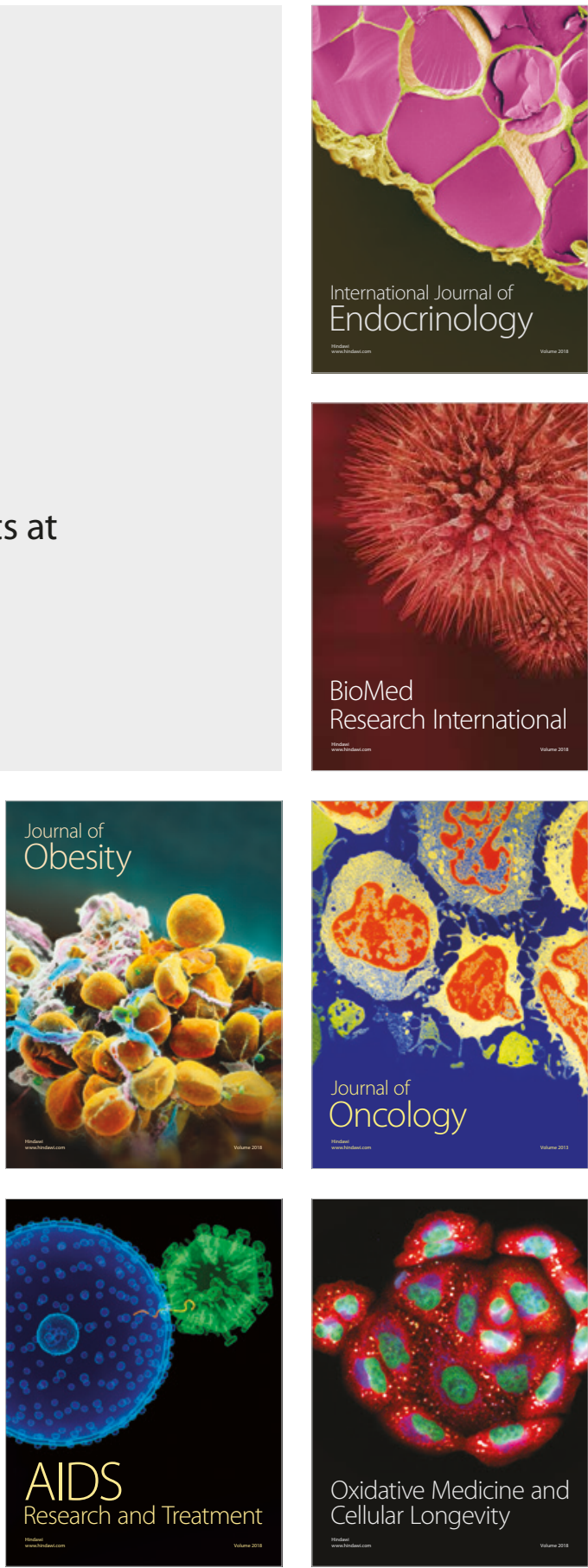